\title{
Study on the change of electron temperature inside magnetic island caused by localized radio frequency heating
}

\author{
J. Yang ${ }^{1,4}$, S. Zhu ${ }^{1,4}$, Q. Yu², and G. Zhuang ${ }^{3}$ \\ ${ }^{1}$ Institute of Plasma Physics, Chinese Academy of Sciences, Hefei, 230031, China \\ ${ }^{2}$ Max-Planck-Institute für Plasmaphysik, EURATOM Association, 85748, Garching, \\ Germany \\ ${ }^{3}$ College of Electrical and Electronic Engineering, Huazhong University of Science \\ and Technology, Wuhan, 430074, China \\ ${ }^{4}$ Center for Magnetic Fusion Theory, Chinese Academy of Sciences, Hefei, 230031, \\ China \\ E-mail: jhyang@ipp.ac.cn
}

The change of the electron temperature inside magnetic island caused by localized radio frequency (rf) heating is studied numerically by solving the two-dimensional energy transport equation, to investigate the dependence of the temperature change on the location and width of the rf power deposition along the minor radius and the helical angle, the island width, and the ratio between the parallel and the perpendicular heat conductivity. Based on obtained numerical results, suggestions for optimizing the island stabilization by localized rf heating are made. 


\section{I . Introduction}

Magnetic islands are often observed in tokamak plasmas. These islands can be driven by an unfavorable plasma current density gradient (a positive tearing mode stability index $\Delta^{\prime}$ ), the perturbed bootstrap current (neoclassical tearing modes) or the electron temperature gradient (drift tearing mode)[1-7]. Sufficiently large magnetic islands have been found to limit the plasma pressure or even to cause disruptions in tokamak experiments[2-6, 8]. Therefore, the stabilization of large islands is an important issue in fusion research. Existing stabilization methods include changing local plasma current density at the island's o-point or around the rational surface by localized rf current drive, or changing local plasma resistivity by localized rf heating in the island region $[9,10]$. Magnetic islands due to neoclassical tearing modes (NTMs) have been successfully suppressed by electron cyclotron current drive (ECCD) on ASDEX Upgrade[11], JT-60[12], and DIII-D[13]. Alternatively, Electron cyclotron resonance heating (ECRH) is also able to reduce the island size or to slow down the island growth on TEXTOR[14], FTU[15], ASDEX Upgrade[15], and T10[16]. To stabilize magnetic islands more efficiently, the modulated technique to deposit the rf power around the island's O-point has been utilized in experiments[11-15]. Theoretical study indicates that the stabilization by ECRH is more effective than that by ECCD for a sufficiently large island[17].

In this paper the change of the electron temperature inside magnetic islands caused by localized rf heating is studied numerically by solving the two-dimensional energy transport equation. We focus on the electron temperature difference between the o-point and x-point of the island, as which is an important parameter measuring the stabilizing effect of localized rf heating on the island. It is well known that a higher electron temperature at the island's o-point, corresponding to a locally lower plasma resistivity or higher plasma current density, is stabilizing for the island. The dependence of the temperature difference on the rf power, the location and the width of the rf power deposition along the minor radius and the helical angle, and the island 
width is studied.

In addition, the effect of the ratio between the parallel and perpendicular heat difusivities, $\chi_{\| /} / \chi_{\perp}$, on the electron temperature difference is also studied. It is known that the heat transport across the magnetic island is affected by the value of $w / w_{c}$, where $w$ is the island width, and $w_{c}=a\left(\chi_{\perp} / \chi_{/ /}\right)^{1 / 4}\left(\varepsilon a n / 8 L_{q}\right)^{-1 / 2}$ is the heat diffusion layer width at the rational surface, $L_{q}=q / q^{\prime}, q$ is the safety factor, $a$ is the plasma minor radius, $\varepsilon=a / R$ is the inverse aspect ratio, and $n$ is the toroidal mode number $[18,19]$. For $\quad w<<w_{c}$, the contribution to the radial transport from the parallel transport along the magnetic field lines is smaller than that from the perpendicular transport. While for $w>>w_{c}$, the electron temperature profile nearly flattens inside the island except in a layer around the island's separatrix. Around the island's x-point this layer has a width about $w_{c}$ along the minor radius and about $w_{c} d w$ along the helical angle $[18,19]$. One therefore expects that the temperature change inside the island caused by the rf heating is affected by the ratio between the parallel and perpendicular heat difusivities.

\section{II . Computational model}

The periodic cylinder geometry is utilized. The magnetic field $\vec{B}$ is defined as

$$
\vec{B}=B_{0 t} \vec{e}_{t}-(n r / m R) B_{0 t} \vec{e}_{\theta}+\nabla \psi \times \vec{e}_{t},
$$

where $\psi$ is the helical flux function, $\vec{e}_{\theta}\left(\vec{e}_{t}\right)$ is the unit vector in the poloidal (toroidal) direction, $\mathrm{m}$ is the poloidal mode number, the subscript 0 denotes the equilibrium quantity, and $\mathrm{r}$ and $\mathrm{R}$ are the minor and major radius.

The equilibrium safety factor is chosen to be the form $q(r)=q_{0} e^{r / L_{q}}$ to have a constant magnetic shear along the minor radius, where $q_{0}=0.45$ and $L_{q}=0.5 a$ are taken. The perturbed $\psi$ in Eq. (1) is assumed to be $\psi_{i}(r)=\psi_{i 0} B_{0 t}(r / a)^{2}(1-r / a)^{2}$ to have a smooth change along the minor radius, being typical for tearing modes [1]. 
The following electron energy transport equation

$$
\frac{3}{2} n_{e} \frac{\partial T_{e}}{\partial t}=n_{e} \nabla \cdot\left(\chi_{/ /} \nabla_{/ /} T_{e}\right)+n_{e} \nabla \cdot\left(\chi_{\perp} \nabla_{\perp} T_{e}\right)+p_{b g}(r)+p_{r f}
$$

is solved, where $T_{e}, n_{e}$ and $p_{r f}$ are electron temperature, density and localized rf power density. $p_{b g}(r)$ is the background heating power density for maintaining the equilibrium electron temperature without the localized rf heating, which includes all other heating or radiation power except the localized rf power. Here $n_{e}, \chi_{/ /}$and $\chi_{\perp}$ are assumed to be constant along the minor radius for simplicity, and the convective transport is neglected.

The rf power density $p_{r f}$ in Eq. (2) is taken to be the form

$$
p_{r f}=p_{r f 0} \exp \left[-\left(\frac{r-r_{e c}}{w_{e}}\right)^{2}\right] \Pi\left(h_{0}, \Delta h\right)
$$

where $p_{r f 0}, r_{e c}$ and $w_{e}$ define the amplitude, the radial location and half-width of $p_{r f}$ respectively. $\Pi\left(h_{0}, \Delta h\right)$ is a square box function given by

$$
\Pi\left(h_{0}, \Delta h\right)=1 \text { for } \quad\left|h-h_{0}\right|<\Delta h
$$

and

$$
\Pi\left(h_{0}, \Delta h\right)=0 \quad \text { elsewhere }
$$

for taking into account the wave deposition profile along the helical angle $h=m \theta+n \phi$, where $\theta$ and $\phi$ are the poloidal and toroidal angle respectively. $h_{0}$ is the helical angle at which the rf power deposition is centered, and $\Delta h$ is the half-width of the rf wave deposition along the helical angle. The boundary conditions used here are $T_{e}(r=a)=0$ and $\frac{d T_{e}(r=0)}{d r}=0$.

\section{Modeling results}

A single $m / n=3 / 2$ magnetic island is considered here. Equation (2) is solved 
numerically in order to obtain the electron temperature change due to the localized rf power. A peaked radial profile of the background heating power density, $p_{b g}(r)=p_{0}\left(1-r^{2}\right)^{8}$, is assumed. The relative electron temperature difference between the o-point and x-point of the magnetic island is defined as

$$
\delta T_{e} \equiv \frac{T_{e(o)}-T_{e(x)}}{T_{e(x)}},
$$

where $T_{e(o)}\left(T_{e(x)}\right)$ is the temperature at island's o-point (x-point).

In tokamak experiments using ECRH to stabilize the magnetic island, the location of the rf power deposition is usually not exactly at the rational surface due to technical limitations. To study the effect of the radial location of rf power deposition on the island stabilization, the values of $\delta T_{e}$ in steady state, obtained by solving Eq. (2), are shown in Fig. 1 as a function of the normalized radial location of rf power deposition, $r_{e c} / a$, for $\log \left(\chi_{1 /} / \chi_{\perp}\right)=5,6,7,8,9$ with $P_{r f} / P=0.084$, where $P$ and $P_{r f}$ is the total background heating power and the total rf heating power, respectively. The island width is $w_{3 / 2}=0.151 a$, and $\varepsilon=a / R=0.3$. The $q=3 / 2$ surface is at $r_{s}=0.601 a$, and the inner and outer edges of the island are at $r_{-}=0.523 a$ and $r_{+}=0.673 a$ respectively, as marked in Fig. 1 by straight vertical lines. The radial rf power deposition width is we=0.01a , and the width along the helical angle is $\Delta \mathrm{h}=0.3 \mathrm{rad}$. $\mathrm{h}_{0}=0$ is taken (corresponding to the helical angle being the same as that of the island's o-point). It is seen that $\delta T_{e}$ has a maximum value when $r_{e c} / a$ is at the rational surface, and it decreases when $r_{e c} / a$ is shifted away from the rational surface as expected. When $r_{e c} / a$ is outside the island, the slightly negative values of $\delta T_{e}$ for $\log \left(\chi_{1 /} / \chi_{\perp}\right)=5,6,7$ is caused by the peaked radial profile of the background heat source[20]. It is also seen from Fig. 1 that the amplitude of $\delta T_{e}$ is affected by the value of $\chi_{1 /} / \chi_{\perp}$. 


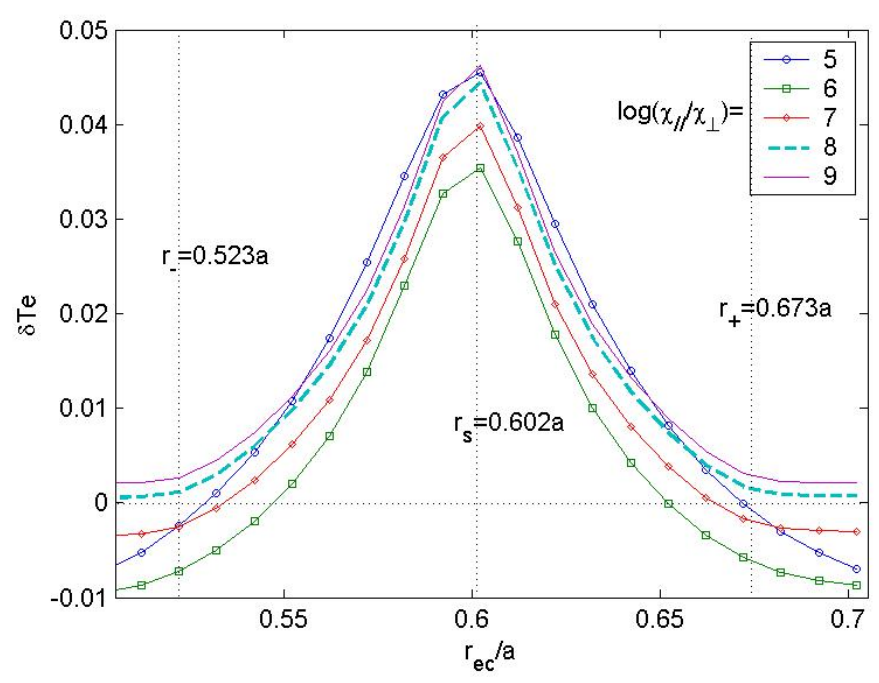

Fig. 1

The higher electron temperature at the island's o-point corresponds to lower plasma resistivity or a higher plasma current density there than those at the island's x-point, which plays a stabilizing role in island growth. Using the Rutherford equation one has[17, 20]

$$
\frac{d w}{d t} \approx \eta\left(\Delta^{\prime}+0.5 \Delta_{\delta T}\right)
$$

where $\quad \Delta_{\delta T}=-\frac{48}{r_{s}} \frac{q}{q^{\prime}} \frac{1}{w} \delta T_{e}$ is contributed from $\delta T_{e}$.

Corresponding to Fig. 1, the values of $\Delta_{\delta T}$ are shown as a function of $r_{e c} / a$ in Fig. 2 for $\log \left(\chi_{1 /} / \chi_{\perp}\right)=6,7,8,10$. When the rf power deposition is at the rational surface, $\Delta_{\delta T}$ has a minimum value about $\Delta_{\delta T} \sim-4$, being of the same order as the low-m tearing mode stability index $\Delta^{\prime}$. One usually has $\left|\Delta^{\prime}\right| \sim m / r_{s}[6,19]$. As the rf power deposition is shifted away from island, $\left|\Delta_{\delta T}\right|$ decreases to about zero in agreement with the TEXTOR experimental observations [14]. 


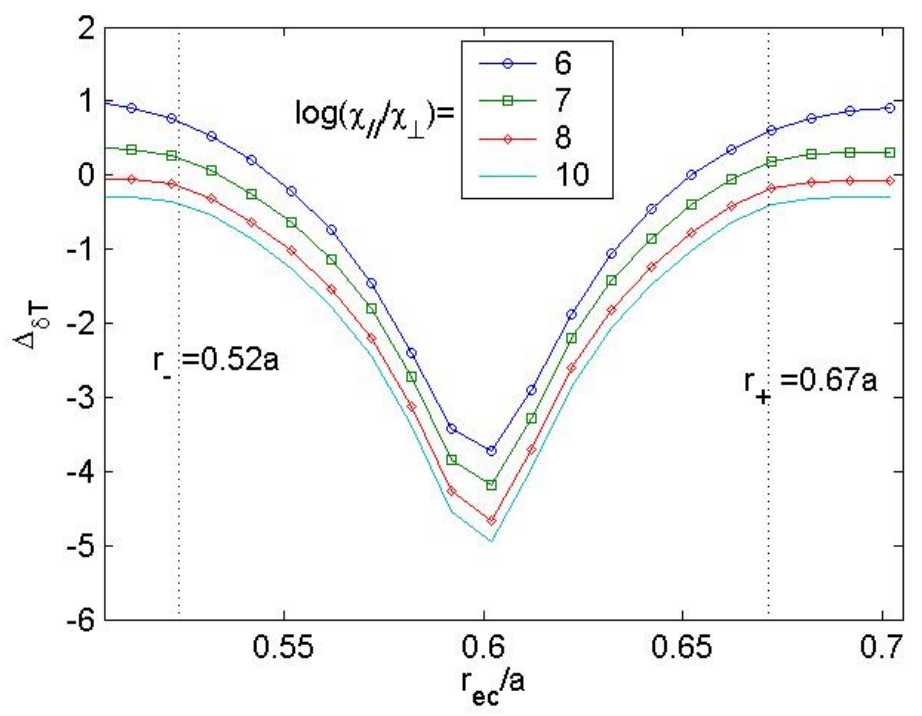

Fig. 2

In Fig. 3 the values of $\delta T_{e}$ are shown as a function of the normalized rf power $P_{r f} / P$ for $\log \left(\chi_{1 /} / \chi_{\perp}\right)=4,5,6,7$, and 10. The center of rf power deposition is taken to be at island's O-point, and other parameters being the same as those of Fig.1. The value of $\delta T_{e}$ increases with the rf power as expected. The increase of $\delta T_{e}$ with $P_{r f}$ is slower than a linear increase because of the change of the background temperature caused by rf power at a constant perpendicular heat difusivity. For the same amount of $P_{r f}$, the values of $\delta T_{e}$ are significant smaller for a higher $\chi_{\| /} / \chi_{\perp}$ than that for a lower $\chi_{\|} / \chi_{\perp}$. This is due to the fast parallel heat transport for a high $\chi_{1 /} / \chi_{\perp}$, which re-distributes the rf power along the magnetic field to a larger region. The heat diffusion layer width are $\mathrm{w}_{\mathrm{c}}=0.145 \mathrm{a}$ and $0.258 \mathrm{a}$ for $\chi_{/ /} / \chi_{\perp}=10^{5}$ and $10^{4}$, respectively, indicating that the fast increase of $\delta T_{e}$ with increasing $P_{r f}$ exists for $\mathrm{w}_{\mathrm{c}}>\mathrm{w}(=0.151 \mathrm{a})$. As the parallel electron heat conductivity is inversely proportional to the electron temperature, localized rf heating is more effective to increase $\delta T_{e}$ for lower temperature plasmas with larger values of $\mathrm{w}_{\mathrm{c}}$, if one assumes that the perpendicular heat difusivity does not significantly change. 


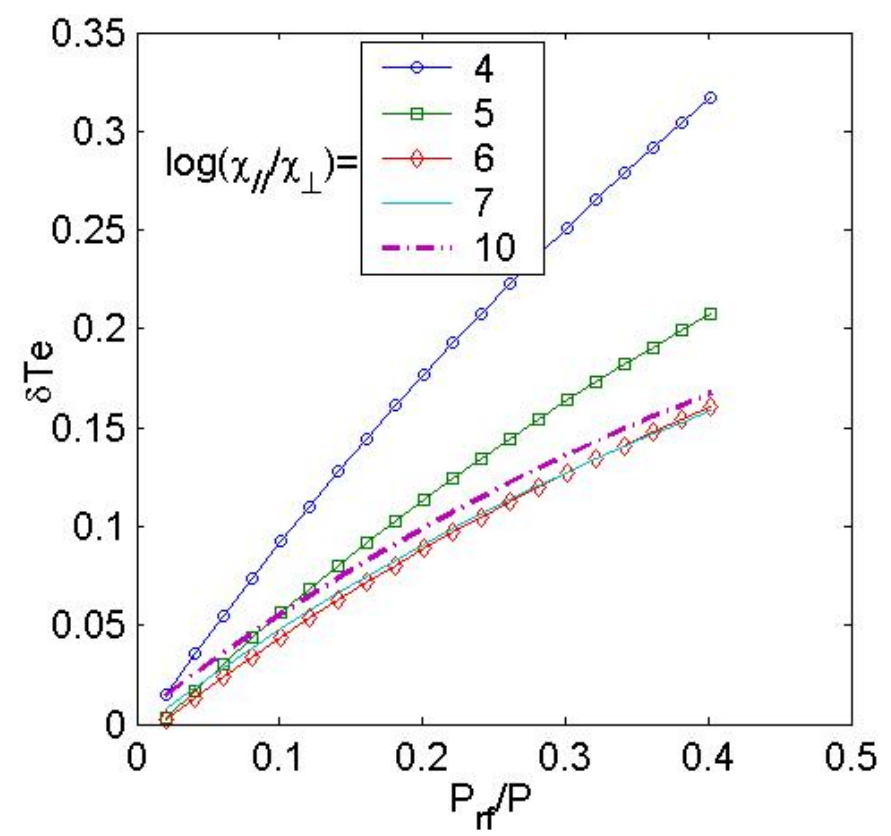

Fig. 3

It is well known that, with continuous increase plasma density, plasma will be eventually subjected to the density limit and disrupted [8, 21]. Before the major disruption, the growth of the $m=2$ magnetic island is observed, followed by a fast drop in the plasma temperature and a slower decay of the plasma current. The local electron temperature at the $q=3$ and 2 surfaces in the current decay phase can be decreased to the range 10-100 eV [21-23]. For tokamak plasmas with $\mathrm{Z}_{\text {eff }}=3$ and $n_{e}=10^{20} \mathrm{~m}^{-3}, \quad \chi_{1 /} / \chi_{\perp}$ equals $5.0 \times 106$ for $\mathrm{T}_{\mathrm{e}}=100 \mathrm{eV}$ and $1.6 \times 104$ for $\mathrm{T}_{\mathrm{e}}=10 \mathrm{eV}$, respectively, by using the classical parallel heat conductivity and assuming $\chi_{\perp}=1 \mathrm{~m}^{2} / \mathrm{s}$. This suggests that localized rf heating can be an effective method to reduce the island width or to slow down the island growth for disruption mitigation, as observed in the experiments[15]. 


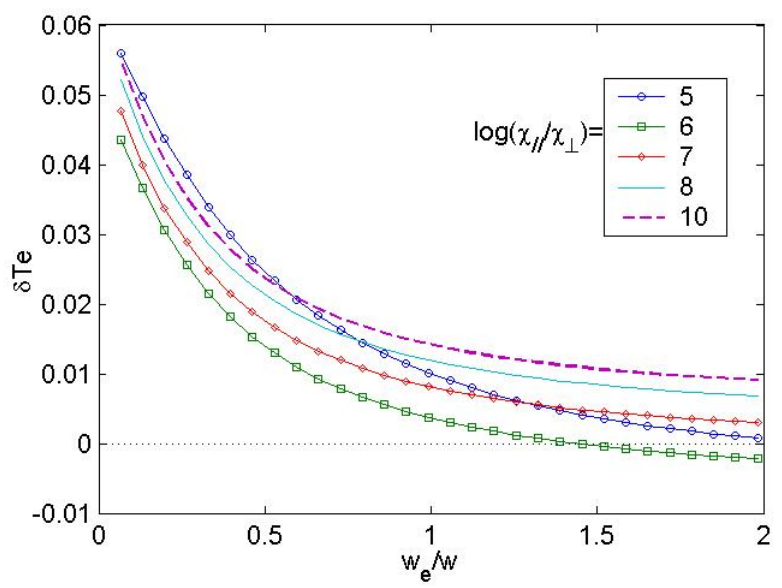

Fig. 4

The values of $\delta T_{e}$ are shown as a function of normalized radial width of $\mathrm{rf}$ power deposition, $w_{e} / w$, in Fig. 4 for $\log \left(\chi_{/ /} / \chi_{\perp}\right)=5,6,7,8,10$ with $P_{r f} / P=0.109$. The other parameters are the same as those of Fig. 3. One finds that for $2 w_{e} / w<1$, i.e., the radial rf power deposition width is smaller than the island width, $\delta T_{e}$ significantly increases with decreasing $\mathrm{w}_{\mathrm{e}}$, indicating a narrow radial rf power deposition is favorable for the island stabilization, as expected.

For a constant perpendicular heat difusivity, the energy confinement time of the island itself is longer for a larger island, leading to a corresponding larger local temperature increase at the same amount of rf power. In Fig.5 values of $\left[T_{e}(o)-T_{e}(x)\right]$ are shown as a function of the normalized island width $w / a$ for $P_{r f} / P=0.2009$ and $\log \left(\chi_{/ /} / \chi_{\perp}\right)=6,7,8,9,10 \quad$, corresponding to $\mathrm{w}_{\mathrm{c}} / \mathrm{a}=0.082$, 0.046, 0.0268, 0.0145, and 0.0082. The other parameters are the same as those of Fig. 3. It is seen that for $w>w_{c}$ the value of $\left[T_{e}(o)-T_{e}(x)\right]$ linearly increase with island width. For $w<w_{c}$, however, it approaches a constant, as in this case the parallel heat transport is not important, and the radial deposition width of rf power is smaller than the island width. Fig. 5 again suggests that localized rf heating is more effective in increasing $\delta T_{e}$ for lower temperature plasmas with larger $\mathrm{w}_{\mathrm{c}}$. 


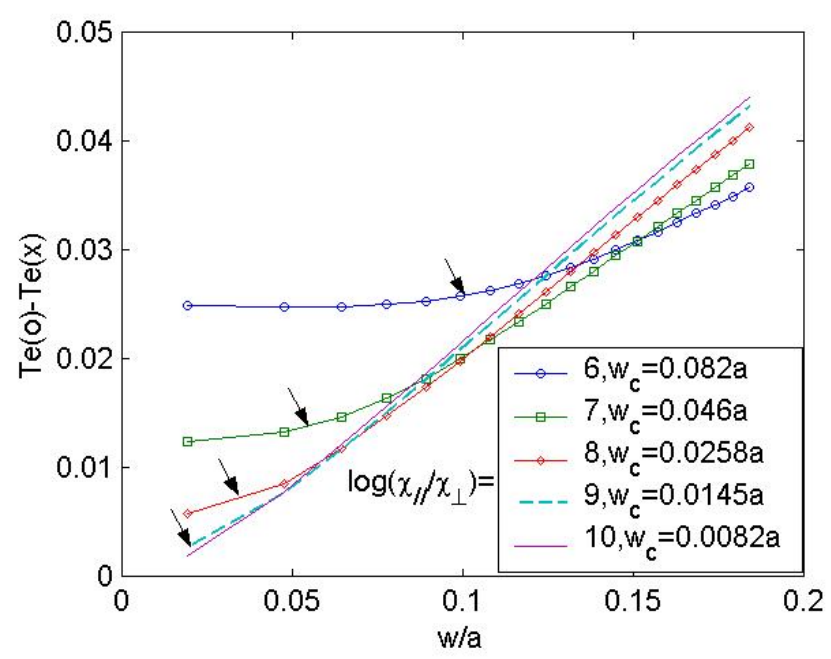

Fig. 5

Above results are obtained for $h_{0}=0$. In tokamak experiments magnetic islands usually rotate in the toroidal direction before mode locking. The rotating island passes through the rf wave deposition region periodically if the radial wave deposition is around the rational surface. In Fig.6 $\delta T_{e}$ is shown as a function of the normalized rf power deposition location along the helical angle, $h_{0} / \pi$, for $\log \left(\chi_{/ /} / \chi_{\perp}\right)=4,5,6,7,8,11, \quad P_{r f} / P=0.0572, \Delta \mathrm{h}=0.1 \mathrm{rad}$, and the other parameters are the same as those of Fig. 3 . Here $h_{0}$ is helical angle at which the rf power density is centered as seen from equations (3)-(5), and $h_{0}=0(\pi)$ corresponds to the rf power deposition at the island's o-point (x-point). $\delta T_{e}$ has a maximum (minimum) value when $h_{0}$ is at island's o-point (x-point), in agreement with TEXTOR experimental results[14]. 


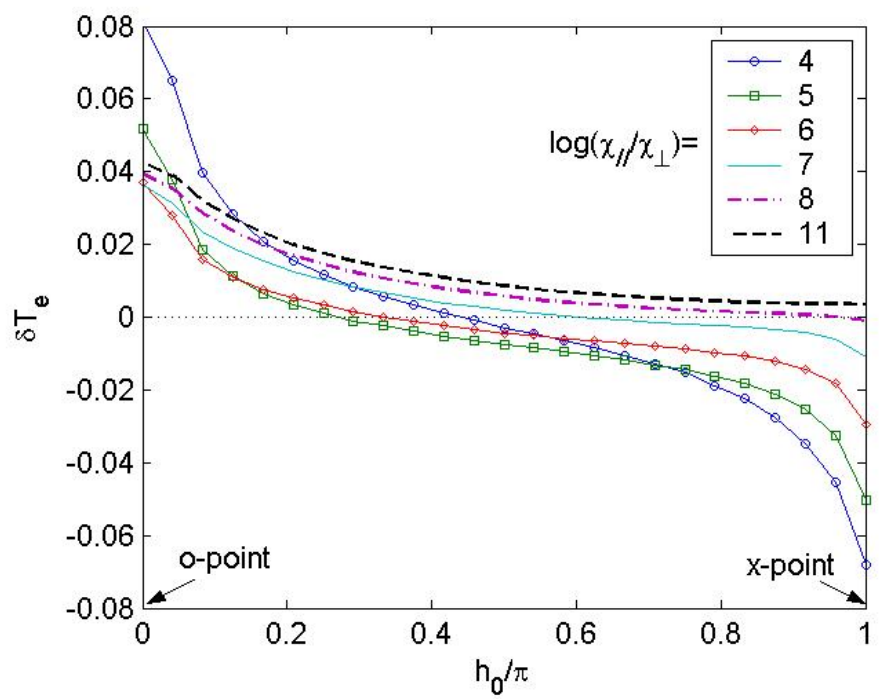

Fig. 6

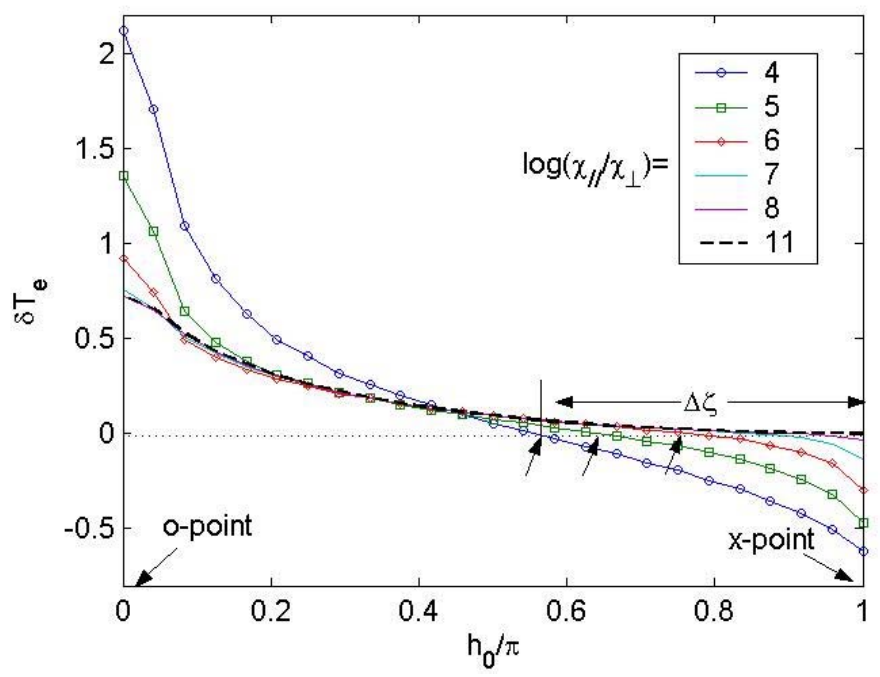

Fig. 7

Fig. 7 is the same as figure 6 except that the background heat source is set to be zero. This allows us to see the effect of the localized rf heating more easily without the additional effect of the background heating power. The value of $h_{0}$ at which $\delta T_{e}=0$ is marked with arrows in the figure, and the helical angle between this location and the island's $\mathrm{x}$-point decreases with increasing $\chi_{/ /} / \chi_{\perp}$.

Fig. 8 is the same as figure 7 except that the value of $\delta T_{e}$ is shown as a function of $\Delta \zeta / \zeta_{c}$, where $\Delta \zeta=\left(\pi-h_{0}\right)$ is the helical angle measured from the x-point, and $\zeta_{c}=w_{c} / w$ is the heat diffusion layer width along the helical angle around the 
island's $\mathrm{x}$-point for $\mathrm{w}>>\mathrm{w}_{\mathrm{c}}[18] . \quad \Delta \zeta=0$ corresponds to the rf power deposition at X-point. It is seen that $\delta T_{e}$ changes from negative to positive value at $\Delta \zeta=0.5 \zeta_{\mathrm{c}}$ for all other curves except for the case with $\log \left(\chi_{1 /} / \chi_{\perp}\right)=5$, for which one has $\mathrm{wc}_{\mathrm{c}}=0.145 \mathrm{a}$ being comparable to the island width.

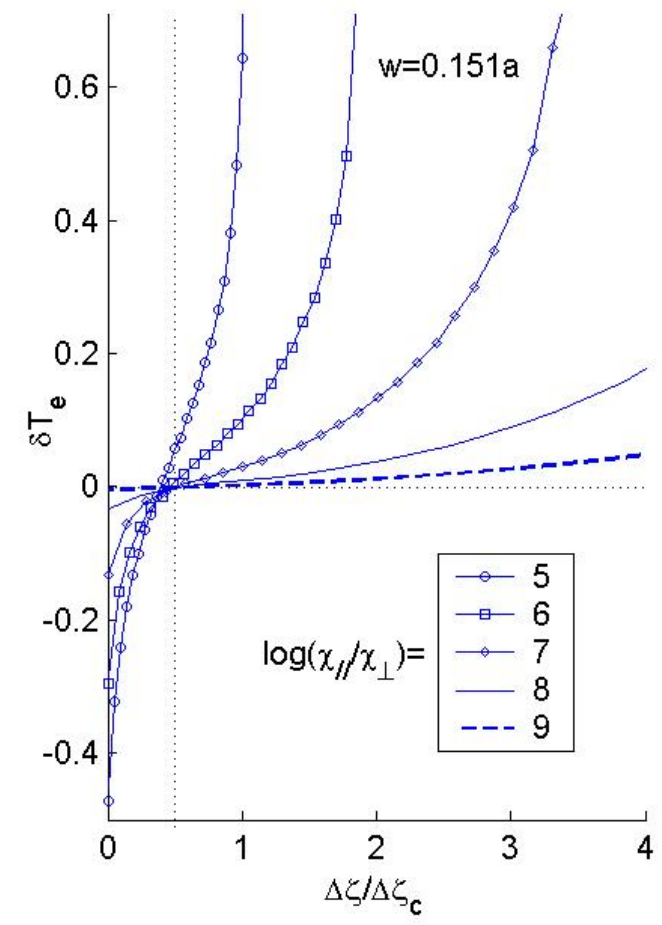

Fig. 8

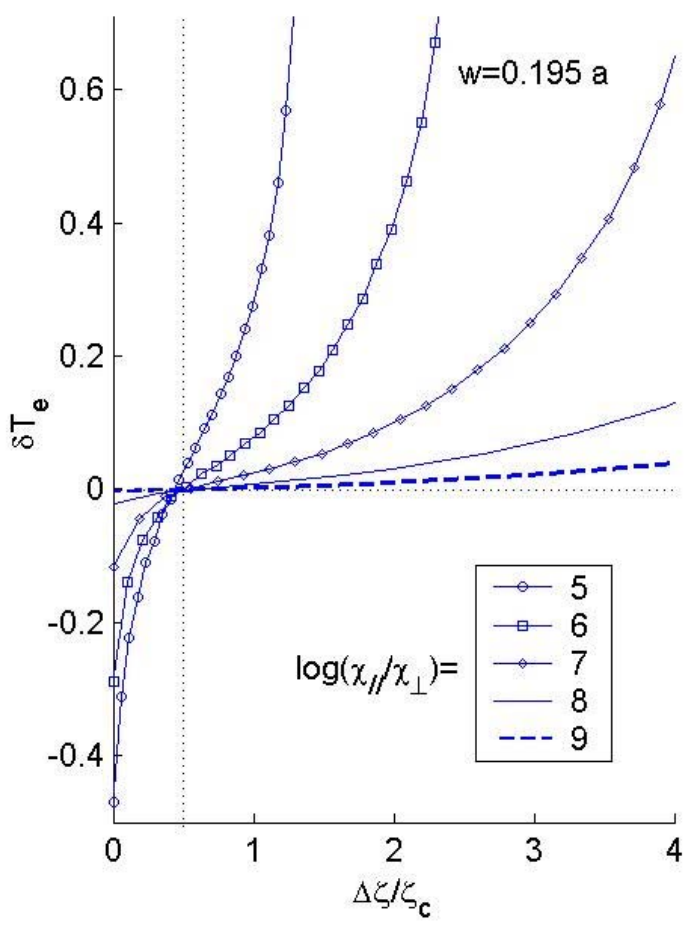

Fig. 9

Being similar to Fig. 8, the value $\delta T_{e}$ is shown as a function of $\Delta \zeta / \zeta_{c}$ in Fig. 9 for a larger island, $w=0.195 a$. In this case $\delta T_{e}$ changes from negative to positive value at $\Delta \zeta=0.5 \zeta_{\mathrm{c}}$ for all curves, as the condition $w>w_{c}$ is satisfied.

In Fig. 10 the value $\delta T_{e}$ is shown as a function of $h_{0} / \pi$ for a small island with $w=0.039 a$. In this case $\mathrm{w}^{<} \mathrm{w}_{\mathrm{c}}$, and $\delta T_{e}$ changes from positive to negative value at $\mathrm{h}_{0}=\pi / 2$, i.e., the middle between the o-point and the x-point. Figs. 7-10 indicate that, in order to optimize the rotating island stabilization by modulated rf heating, the helical angle for rf power deposition should be chosen based on the value of $w_{d} d w$. 


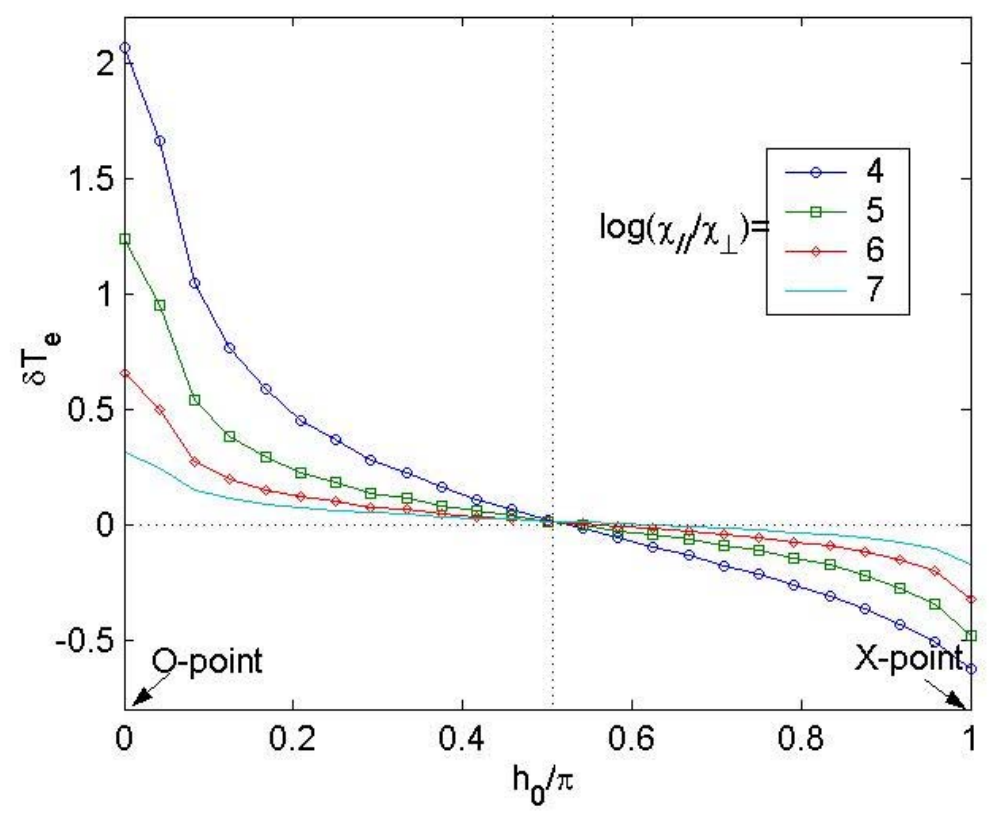

Fig. 10

On the other hand it is well known that, once the island is large enough, it will be locked by the machine error field or the helical current induced in the vacuum vessel [21-24]. After the island's o-point is locked at a particular toroidal and poloidal angle by the intrinsic machine error field, it is not necessarily covered by rf wave deposition. It was found recently that a large island can be locked by a small amplitude helical field in slowly rotating plasmas, while the island size is essentially not affected [24]. This suggests that an actively applied helical field can be utilized to control the location of the island's o-point to be in the rf wave deposition region, to enable the island stabilization by rf heating after mode locking. In this case additional optimization could result from a narrow rf power deposition width along the helical angle, as shown in Fig. 11, in which $\delta T_{e}$ is shown as a function of normalized rf power deposition width along the helical angle, $\Delta h / \pi$, for $\log \left(\chi_{1 /} / \chi_{\perp}\right)=4,5,6,7,10$ with $P_{r f} / P=0.261$ and the other parameters being the same as those of Fig.3. It is seen that with decreasing $\Delta h / \pi, \delta T_{e}$ significantly increases. The increase is much larger for a lower $\chi_{/ /} / \chi_{\perp}$ (larger wc), suggesting that a narrow rf power deposition width along the helical angle is very favorable for stabilizing locked islands, if the 
island is large enough to be locked by a small amplitude helical field in the desired phase.

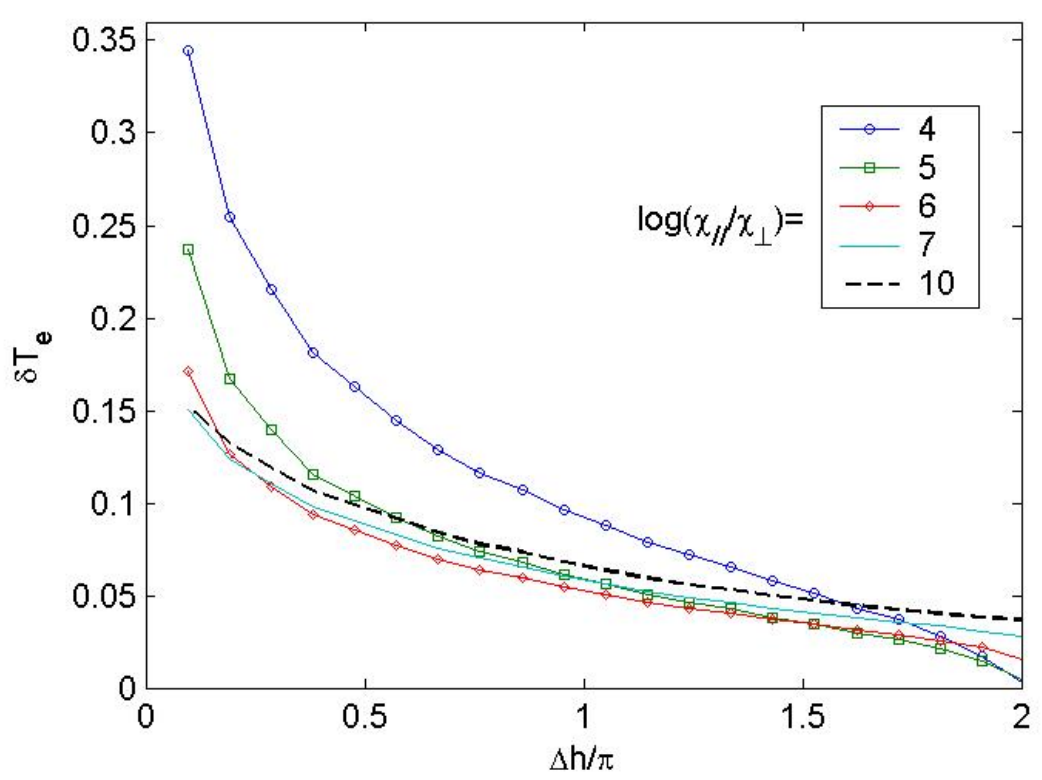

Fig. 11

\section{Discussion and Summery}

Our results show that, in order to efficiently increase the electron temperature inside the magnetic island by localized rf heating, the radial location of the rf power deposition should be as close to the rational surface as possible, and the radial deposition width of rf power should be as narrow as possible, as expected and seen from experimental results[11-16].

For rotating magnetic islands usually observed in tokamak experiments, the modulated technique to deposit the rf power around the island's O-point is obviously better than a non-modulated one. The optimized helical angle for the modulation is determined by the ratio between the island width and the heat diffusion layer width, $\mathrm{w} / \mathrm{w}_{\mathrm{c}}$. For $w<<w_{c}$, the half duty cycle for the modulation leads to a highest stabilizing efficiency as seen from Fig. 10. With increasing w/wc, the optimized helical angle for rf power deposition extends towards the x-point. The value of $w_{c}$ depends on plasma parameters, as $w_{c} \sim\left(\chi_{\perp} / \chi_{1 /}\right)^{1 / 4} \sim T_{e}^{-5 / 8} n_{e}^{1 / 4} \chi_{\perp}^{1 / 4}$ by using the 
classical parallel heat conductivity. During the island growth from a small amplitude, the value of $\mathrm{w} / \mathrm{w}_{\mathrm{c}}$ increases accordingly. Sufficiently large islands, however, could result in significant drop in the electron temperature in the island region, as seen in tokamak experiments, e.g., when the plasma density is close to the density limit[21-23], leading to a corresponding increase in $\mathrm{w}_{\mathrm{c}}$. To efficiently stabilize the island, the helical angle for rf power modulation should be taken based on the value of $\mathrm{w} / \mathrm{w}_{\mathrm{c}}$.

Stabilizing of the large island by ECCD observed before disruption is not efficient because of the low electron temperature, leading to a low rf current drive efficiency. The localized rf heating, however, has a higher stabilizing efficiency in this case due to the lower value of $\chi_{/ /} / \chi_{\perp} \quad\left(\right.$ larger $\mathrm{w}_{\mathrm{c}}$ ) as seen from Figs. 3 and 5 . In addition, the effect of rf heating increases with the island width. This suggests that localized rf heating is a possible method to stabilize a large island or to slowing down the island growth for disruption mitigation.

The validity of Eq. (2) and the constant $\chi_{\|}$assumption in our calculations should be discussed. It was shown by the analytical theory that, the classical heat conductivity $\chi_{\| c}$ is valid only for $\mathrm{k}_{\|} \lambda_{\mathrm{e}}<1$. While for $\mathrm{k}_{\|} \lambda_{\mathrm{e}}>1, \chi_{\|} \approx \mathrm{v}_{\mathrm{Te}} / \mathrm{k}_{\|}$due to the "heat flux limit”, where $\lambda_{e}$ is the electron mean-free path, $\mathrm{v}_{\mathrm{Te}}$ is the electron thermal velocity, $\mathrm{k}_{\|}=\mathbf{B}_{0} \cdot \mathbf{k} /\left|\mathbf{B}_{0}\right|$, and $\mathbf{k}$ is the wave vector of the island [25, 26]. In the lowest order $k_{\|}=n \mid r-r_{s} /\left(L_{q} R\right)$, where $r_{s}$ is the minor radius of the rational surface. For tokamak plasmas with $\mathrm{a}=1 \mathrm{~m}, \mathrm{a} / \mathrm{R}=0.3, \mathrm{Lq}=0.5 \mathrm{a}, \mathrm{Z}_{\mathrm{eff}}=3, \mathrm{~T}_{\mathrm{e}}=500 \mathrm{eV}$, and $n_{e}=10^{20} \mathrm{~m}^{-3}$, the condition $\mathrm{k}_{\|} \lambda_{\mathrm{e}}<1$ leads to $\left|\mathrm{r}-\mathrm{r}_{\mathrm{s}}\right|<0.18 \mathrm{a}$. For an island with its width $\mathrm{w}<0.18 \mathrm{a}$, across the island region $\chi_{\|}$is still by $\chi_{\| c}$. Since $T_{e}$ changes little in the island region, the constant $\chi_{\|}$assumption is reasonable. In the outer region away from the island one has $\mathrm{k}_{\|} \lambda_{\mathrm{e}}>1$. In this region, however, the use of $\chi_{\|} \approx \mathrm{v}_{\mathrm{Te}} / \mathrm{k}_{\|}$or $\chi_{\| \mathrm{c}}$ will lead to the same result: the temperature along the field lines becomes a constant due to the fast 
parallel transport, since both forms of $\chi_{\|}$are large enough to lead to $\chi_{\|} / \chi_{\perp}>>1$. Therefore, for a low temperature and high density tokamak edge plasma being closing to the density limit, the use of $\chi_{\| \mathrm{c}}$ leads to the correct results both inside and outside the island. As $\lambda_{\mathrm{e}} \sim \mathrm{T}_{\mathrm{e}}{ }^{2} / \mathrm{n}_{\mathrm{e}}$, for a high temperature or low density plasma, however, the classical heat conductivity is valid in a much smaller region around the rational surface or the island's x-point. Away from this region the heat flux is carried by free-streaming electrons $[25,26]$. In this case the "flux limit" approximation for $\chi_{1 /}$ is equivalent to replacing conduction by convection $n_{e} v_{T e} \nabla_{/ /} T_{e}$ in the electron energy transport equation. In the present paper the complicated physics of the parallel heat flux has not be addressed. Future calculations with a more exact model for the parallel heat transport is necessary for high temperature (or low density) plasmas. As the "flux limit" approximation for $\chi_{/ /}$leads to a much smaller $\chi_{/ /}$than that of the classical value in this case, the localized rf heating is expected to have a stronger stabilizing effect due to the lower value of $\chi_{\|} / \chi_{\perp}$ as seen from Figs. 3 and 5.

It should be mentioned that the change of the tearing stability index $\Delta^{\prime}$ by the rf heating has not been considered here. Future studies using self-consistent radial profiles of the plasma current density and the electron temperature and simultaneously calculating both the magnetic and temperature perturbations are still required to further study the effect of the localized rf heating on the island.

In summary, the relative electron temperature difference between the island's o-point and x-point caused by localized rf heating is studied. It is found that:

(1) For a given amount of rf power, the temperature difference is larger for a larger island and a lower ratio between the parallel and the perpendicular heat conductivity, suggesting that localized rf heating is an effective way for stabilizing large islands before disruptions in agreement with experimental observations[15].

(2) To stabilize rotating islands more efficiently, the optimized helical angle for the rf power modulation is determined by the ratio between the island width and the 
heat diffusion layer width, $\mathrm{w}_{\mathrm{w}} \mathrm{w}_{\mathrm{c}}$. For $w>w_{c}$, the rf power should not be deposited in the region around the island's x-point with a width $\zeta_{c} \sim w_{c} / w$ along the helical angle. For $w<w_{c}$, however, the half duty cycle for the modulation has the highest stabilizing efficiency.

(3) If an applied helical field is utilized to control the location of a locked island's o-point to be in the rf wave deposition region, the stabilizing effect significantly increases with decreasing rf power deposition width along the helical angle.

\section{ACKNOWLEDGEMENT}

This work is supported by the Chang-Jiang scholar project of the Ministry of Education of China. The authors would like to thank the Center for Computational Science, Hefei Institute of Physical Sciences for its support.

\section{Reference:}

[1] H.P.Furth, J.Killen, and M.N.Rosenbluth, Phy.Fluids 6, 459(1963).

[2] Z.Chang, J.D.Callen, E.D.Fredrickson, R.V.Budny, C.C.Hegna, K.M.McGuire, M.C.Zarnstorff, and TFTR group, Phys. Rev. Lett. 74, 4663(1995).

[3] H.Zohm, G. Gantenbein, A. Gude, S. Günter, F. Leuterer, M. Maraschek, J. Meskat, W. Suttrop, Q. Yu, ASDEX Upgrade Team, and ECRH-Group (AUG), Phys. Plasmas 8, 2009(2001).

[4] S.Günter, A. Gude, M. Maraschek, S. Sesnic, H. Zohm, ASDEX Upgrade Team, and D. F. Howell, Phys. Rev. Letter $\quad$ 87, 275001(2001).

[5] R.J.L.Haye, L. L. Lao, E. J. Strait, and T. S. Taylor, Nucl. Fusion 37, 397(1997).

[6] O.Sauter, R. J. L. Haye, Z. Chang, D. A. Gates, Y. Kamada, H. Zohm, A. Bondeson, D. Boucher, J. D. Callen, M. S. Chu, T. A. Gianakon, O. Gruber, R. W. Harvey, C. C. Hegna, L. L. 
Lao, D. A. Monticello, F. Perkins, A. Pletzer, A. H. Reiman, M. Rosenbluth, E. J. Strait, T. S. Taylor, A. D. Turnbull, F. Waelbroeck, J. C. Wesley, H. R. Wilson, and R. Yoshino, Phys. Plasmas 4, 1654(1997).

[7] Q.Yu, S. Günter, and B. D. Scott, Phys. Plasmas 10, 797(2003).

[8] J.Wesson, Tokamaks Third edition, edited by J. Birman, et al. (Clarendon Press,Oxford,1987).

[9] H. Zohm, Phys. Plasmas 4, 3433(1997).

[10] C.C.Hegna and J. D. Callen, Phys. Plasmas 4 2940(1997).

[11] G.Gantenbein, H. Zohm, G. Giruzzi, S. Günter, F. Leuterer, M. Maraschek, J. Meskat, Q. Yu, ASDEX Upgrade Team, and ECRH-Group (AUG), Phys. Rev. Lett. 85, 1242(1999).

[12] A.Isayama, G. Matsunaga, T. Kobayashi, S. Moriyama, N. Oyama, Y. Sakamoto, T. Suzuki, H. Urano, N. Hayashi, Y. Kamada, T. Ozeki, Y. Hirano, L. Urso, H. Zohm, M. Maraschek, J. Hobirk, K. Nagasaki, and the JT-60 team, Nucl. Fusion 49, 055006 (2009).

[13] C.C.Petty, R. J. L. Haye, T. C. Luce, D. A. Humphreys, A. W. Hyatt, J. Lohr, R. Prate, E. J. Strait, and M. R. Wade, Nucl. Fusion 44243 (2004).

[14] E.Westerhof, A.Lazaros, E.Farshi, M.R.de Baar, M.F.M.de Bock, I.G.J.Classen, R.J.E.Jaspers, G.M.D.Hogeweij, H.R.Koslowski, A.Kraemer-Flecken, Y.Liang, N.J.Lopes Cardozo, and O.Zimmermann, Nucl.Fusion 47,85(2007).

[15] B.Esposito, G. Granucci, S. Nowak, J. R. Martin-Solis, L. Gabellieri, E. Lazzaro, P. Smeulders, M. Maraschek, G. Pautasso, J. Stober, W. Treutterer, L. Urso, F. Volpe, H. Zohm, and FTU ECRH and ASDEX Upgrade Teams, Nucl. Fusion 49065014 (2009)

[16] K.A.Razumova, Plasma Phys. Report 27 273(2001).

[17] Qingquan Yu and Sibylle Günter, Plasma Phys. Control. Fusion 40 1977(1998)

[18] Q.Yu, Phys. Plasmas 13, 062310(2006).

[19] R.Fitzpatrick, Phys. Plasmas 2, 825(1995).

[20] J.Yang, Q. Yu, S. Zhu, and G. Zhuang, Phys. Plasmas 16, 92308 (2009).

[21] J.A.Wesson, R. D. Gill, M. Hugon, F. C. Schuller, J. A. Snipes, D. J. Ward, D. V. Bartlett, D. J. Campbell, P. A. Duperrex, A. W. Edwards, R. S. Granetz, N. A. O. Gopttardi, T. C. Hender, E. Lazzaro, P. J. Lomas, N. Lopes Cardozo, K. F. Mast, M. F. F. Nave, N. A. Salmon, P. Smeulders, P. R. Thomas, B. J. D. Tubbing, M. F. Turner, and A. Weller, Nucl. Fusion 29, 641 (1989).

[22] W.Suttrop, K. Buchl, J. C. Fuchs, M. Kaufmann, K. Lackner, M. Maraschek, V. Mertens, R. Neu, M. Schittenhelm, M. Sokoll, and H. Zohm, Nucl. Fusion 37 119(1997).

[23] D.J.Ward and J. A. Wesson, Nucl. Fusion 32, 1117(1992).

[24] Q.Yu and S. Günter, Nucl. Fusion 48, 065004(2008).

[25] Z.Chang and J.D.Callen, Phys. Fluids B 4, 1167(1992).

[26] G.W.Hammett and F. W. Perkins, Phys. Rev. Lett. 64, 3019(1990).

\section{CAPTION}

Figure 1 (color online) $\quad \delta T_{e}$ is shown as a function of the normalized radial location of rf power deposition, $r_{e c} / a$, for $\log \left(\chi_{\| \prime} / \chi_{\perp}\right)=5,6,7,8,9$ with $\mathrm{w}_{\mathrm{e}}=0.01 \mathrm{a}$, $\mathrm{h}_{0}=0, \Delta \mathrm{h}=0.3 \mathrm{rad}$, and $P_{r f} / P=0.084$. The island width $w_{3 / 2}=0.151 a$. The rational 
surface and the inner and outer edges of the island are marked by straight vertical lines. $\delta T_{e}$ has a maximum value when $r_{e c} / a$ is at the rational surface, and it decreases when $r_{e c} / a$ is shifted away from the rational surface.

Figure 2 (color online) Corresponding to Fig. 1, $\Delta_{\delta T}$ are shown as a function of $\left(r_{e c} / a\right)$ for $\log \left(\chi_{\| /} / \chi_{\perp}\right)=6,7,8,10$. When $r_{e c} / a$ is at the rational surface, $\Delta_{\delta T}$ has a minimum value. As the rf power deposition is shifted away from island, $\Delta_{\delta T}$ approaches zero.

Figure 3 (color online) $\quad \delta T_{e}$ is shown as a function of the normalized rf power $P_{r f} / P$ for $\log \left(\chi_{1 /} / \chi_{\perp}\right)=4,5,6,7$, and 10 . The center of rf power deposition is at island's O-point, and other parameters are the same as those of Fig.1. $\delta T_{e}$ is significant smaller for a higher $\chi_{1 /} / \chi_{\perp}$ than that for a lower $\chi_{/ /} / \chi_{\perp}$.

Figure 4 (color online) $\quad \delta T_{e}$ is shown as a function of normalized radial width of the rf power deposition, $w_{e} / w$, for $\log \left(\chi_{/ /} / \chi_{\perp}\right)=5,6,7,8,10$ with $P_{r f} / P=0.1086$. The other parameters are the same as those of Fig. 3. For $2 w_{e} / w<1$, i.e., the radial rf power deposition width is smaller than the island width, $\delta T_{e}$ significantly increases with decreasing $w_{e} / w$.

Figure 5 (color online) $\quad T_{e}(o)-T_{e}(x)$ is shown as a function of the normalized island width $w / a$ for $P_{r f} / P=0.2009$ and $\log \left(\chi_{1 /} / \chi_{\perp}\right)=6,7,8,9,10$, with the other parameters being the same as those of Fig. 3. For $w>w_{c}$ the value of $T_{e}(o)-T_{e}(x)$ linearly increase with island width. For $w<<w_{c}$ it approaches a constant.

Figure 6 (color online) $\quad \delta T_{e}$ is shown as a function of the normalized rf power deposition location along the helical angle, $\mathrm{h}_{0} / \pi$, for $\log \left(\chi_{\|} / \chi_{\perp}\right)=4,5,6,7,8,11$, $P_{r f} / P=0.0572, \Delta \mathrm{h}=0.1 \mathrm{rad}$, and the other parameters being the same as those of Fig. 
3. $h_{0}=0(\pi)$ corresponds to the rf power deposition at the island's o-point (x-point). $\delta T_{e}$ has a maximum (minimum) value when $h_{0}$ is at island's o-point (x-point). Figure 7 (color online) Same as figure 6 except that the background heat source is set to be zero. The value of $h_{0}$ at which $\delta T_{e}=0$ is marked with arrows in the figure, and the helical angle between this location and the island's x-point decreases with increasing $\chi_{/ /} / \chi_{\perp}$.

Figure 8 (color online) Same as figure 7 except that $\delta T_{e}$ is shown as a function of $\Delta \zeta / \zeta_{c}$, where $\Delta \zeta=\left(\pi-h_{0}\right)$ is the helical angle measured from the x-point, and $\zeta_{c}=w_{c} / w$ is the heat diffusion layer width along the helical angle around island $\mathrm{x}$-point for $\mathrm{w}>\mathrm{w}_{\mathrm{c}}$. $\delta T_{e}$ changes from negative to positive value at $\Delta \zeta=0.5 \zeta_{c}$ for all other curves except for the case with $\log \left(\chi_{1 /} / \chi_{\perp}\right)=5$, for which $\mathrm{w}_{\mathrm{c}}=0.145$ being comparable to the island width.

Figure 9 (color online) Being similar to Fig. 8, $\delta T_{e}$ is shown as a function of $\Delta \zeta / \zeta_{c}$ for a larger island, $w=0.195 a . \delta T_{e}$ changes from negative to positive value at $\Delta \zeta=0.5 \zeta_{c}$, because $w>w_{c}$ is satisfied for all curves.

Figure 10 (color online) $\delta T_{e}$ is shown as a function of $h_{0} / \pi$ for a small island, $w=0.039 a$. Because $\mathrm{w}<\mathrm{w}_{\mathrm{c}}$ in this case, $\delta T_{e}$ changes from positive to negative value at $\mathrm{h}_{0}=\pi / 2$, the middle between the o-point and the $\mathrm{x}$-point.

Figure 11 (color online) $\quad \delta T_{e}$ is shown as a function of normalized rf power deposition width along the helical angle, $\Delta h / \pi$, for $\log \left(\chi_{1 /} / \chi_{\perp}\right)=4,5,6,7,10$, with $P_{r f} / P=0.261$ and the other parameters being the same as those of Fig.3. With decreasing $\Delta h, \delta T_{e}$ significantly increases, suggesting a narrow rf power deposition width along the helical angle is very favorable for stabilizing locked islands. 